\title{
First Nationwide Survey of the Prevalence of TB/HIV Co-Infection in Ghana
}

\author{
Kennedy K. Addo ${ }^{*}$, William K. Ampofo ${ }^{2}$, Richard Owusu' ${ }^{1}$, Christian Bonsu ${ }^{1}$, Naomi Nartey ${ }^{1}$,

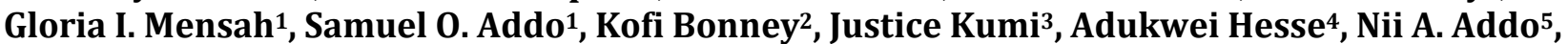 \\ Frank A. Bonsu' ${ }^{6}$
}

\author{
${ }^{1}$ Department of Bacteriology, Noguchi Memorial Institute for Medical Research, Accra, Ghana \\ ${ }^{2}$ Department of Virology, Noguchi Memorial Institute for Medical Research, Accra, Ghana \\ ${ }^{3}$ Department of Clinical Pathology, Noguchi Memorial Institute for Medical Research, Accra, Ghana \\ ${ }^{4}$ Executive Health Care and Consult, Accra, Ghana \\ ${ }^{5}$ National AIDS/STI Control Programme, Accra, Ghana \\ ${ }^{6}$ National Tuberculosis Control Programme, Accra, Ghana \\ Email: *kaddo@noguchi.ug.edu.gh
}

\begin{abstract}
How to cite this paper: Addo, K.K., Ampofo, W.K., Owusu, R., Bonsu, C., Nartey, N., Mensah, G.I., Addo, S.O., Bonney, K., Kumi, J., Hesse, A., Addo, N.A. and Bonsu, F.A. (2018) First Nationwide Survey of the Prevalence of TB/HIV Co-Infection in Ghana. Journal of Tuberculosis Research, 6 , 135-147.

https://doi.org/10.4236/jtr.2018.62013
\end{abstract}

Received: April 5, 2018

Accepted: June 11, 2018

Published: June 14, 2018

Copyright $\odot 2018$ by authors and Scientific Research Publishing Inc. This work is licensed under the Creative Commons Attribution International License (CC BY 4.0).

http://creativecommons.org/licenses/by/4.0/ (c) (i) Open Access

\begin{abstract}
Background: To better understand the extent of the magnitude of tuberculosis (TB) and Human Immunodeficiency Virus (HIV) co-infection in Ghana, a baseline study was conducted to establish the national prevalence of the dual infection. The study aimed to determine the most prevalent HIV serotype (HIV-1 or HIV-2) in TB patients (new and old cases); genotype mycobacterial species causing TB/HIV co-infection and determine their drug susceptibility patterns. Methods: Sputum and dried blood samples were collected from 503 TB patients from 67 health facilities nationwide between December 2007 and November 2008. All samples were processed for mycobacterial and HIV testing using conventional and molecular methods. Results: A total of 517 paired sputum samples were received from 517 patients. A total 503 patients [335 (66.6\%) males; 168 (33.4\%) females] had at least one culture positive sample. Majority (93.0\%) of the patients were new cases while $7.0 \%$ were old cases. All 503 TB isolates were Mycobacterium tuberculosis complex. Of 503 blood samples, 74 were positive for HIV (14.7\%), comprising $71(14.1 \%)$ and 3 (0.6\%) for HIV-1 and HIV-1 \& 2 respectively; none was positive for HIV-2 alone. The seroprevalence of HIV in newly diagnosed TB patients and those already on treatment, was $69 / 468(14.7 \%)$ and $5 / 35(14.3 \%)$ respectively ( $p$ > 0.05). Differentiation of isolates from TB/HIV co-infected patients showed that 70/74 (94.6\%) were Mycobacterium tuberculosis while 4/74 (5.4\%) were Mycobacterium africanum. Monoresistance to isoniazid and rifampicin were 4/74 (5.4\%) and 1/74 (1.4\%) respectively; resistance to both drugs (multi-drug resistant-MDR) was not observed. Sixty nine (93.2\%) isolates were susceptible to both drugs. Conclusion: The prevalence of HIV infection in TB patients
\end{abstract}


was $14.7 \%$. TB/HIV was common among the sexually active age group (25 34 years). Majority of the TB isolates were $M$. tuberculosis which were susceptible to both isoniazid and rifampicin. HIV-1 was the common serotype infecting TB patients in Ghana.

\section{Keywords}

TB/HIV, Co-Infection, Mycobacterium tuberculosis complex, Drug Resistance, Ghana

\section{Introduction}

The syndemic relationship between tuberculosis (TB) and human immunodeficiency virus (HIV) infection has contributed to high morbidity and mortality worldwide [1]. The World Health Organization (WHO) Africa region is the hardest hit where about $74 \%$ of the estimated 1.2 million TB patients co-infected with HIV occurred in 2014 [2]. For many years, efforts to tackle TB and HIV have been largely independent of each other, despite their overlapping epidemiology. The risk of progression from latent to active TB is increased by infection with HIV [3]. Likewise, TB is the most important opportunistic disease that increases progression to acquired immune deficiency syndrome (AIDS) and the number one killer in HIV-positive individuals [4] [5]. Thus effective TB control can contribute to better HIV/AIDS control by reducing the TB burden in people with HIV as well as providing an entry point to HIV prevention and care for people with TB [6]. The HIV prevalence in the general population in Ghana was $1.47 \%$ in 2014 which is an indication of a downward trend over a fifteen-year period from 2.3\% in 2000 [7]. Studies in Africa and Asia have reported high HIV prevalence in TB patients than are observed in the general population [8]. Routine programme data from TB/HIV sites showed that in 2005, 40 percent of TB patients who were tested for HIV were HIV-positive and in 2006 these went down to 33 percent [9] [10]. Ghana is a high burden TB/HIV country [11], however very few studies have reported on the prevalence of TB/HIV co-infection in Ghana [12] [13] [14]. These previous studies were single hospital-based in the two big cities in Ghana-Accra and Kumasi. There was the need to conduct a nationwide study to establish the national prevalence of this dual infection. This study was therefore conducted to determine the prevalence of HIV in TB patients in Ghana.

\section{Materials and Methods}

\subsection{Study Design}

The study was cross-sectional in accordance with WHO Guidelines for HIV surveillance among TB patients [15].

\subsection{Study Population and Sampling Strategy}

All TB patients diagnosed within the study period between December 2007 and 
November 2008 in selected health facilities were eligible for participation. Using a list of all health facilities in the country, the number of registered TB patients in the treatment centres per year was noted. A cumulative population list was then compiled from which participating facilities were selected from all the ten regions of Ghana. Thirty clusters consisting of 67 health facilities were selected and grouped into northern and southern sectors (Table 1). Selection of study participants was done using the consecutive sampling method to minimize bias. Hence, every patient who met the eligibility criteria was selected and after obtaining his/her informed consent was interviewed using a structured questionnaire.

\subsection{Sample Size Calculation}

The minimum sample size was calculated using the formula: $N=P Q /(E / Z)^{2}$

[15] where $N=$ the minimum sample size required; $P=$ the maximum expected prevalence rate; $Q=100-P ; E=$ the margin of sampling error tolerated; $Z=$ the centile of the standard normal

If the confidence level is chosen at $95 \%$ then $Z=1.96$

With the expected HIV prevalence rate among TB patients $(P)$ at $30 \%$

$Q$ will be $100-30=70$; and margin of error at $5 \%$, the minimum sample size was:

$$
N=30 \times 70 /(5 / 1.96)^{2}=2100 / 6.51=323
$$

To allow for losses, a $60 \%$ adjustment was included: $323 \times 60 \%=194$

(These losses included patients diagnosed as having TB who did not return to the centres and from whom it was not possible to obtain 2 sputum samples, patients whose cultures were contaminated or did not grow and patients who refused HIV testing after counselling). Maximum Sample Size $=323+194=517$

\subsection{Sample Collection and Transport}

For each eligible patient, an equal volume of $1 \%$ Cetylpyridinium Chloride (CPC) was added to two sputum samples for homogenization and decontamination on transit and to maintain the viability of the bacilli if present. Blood was collected onto blood spot collection card to prepare dried blood spot according to the study specific protocol [16]. All the samples were stored at $4{ }^{\circ} \mathrm{C}$ and transported within one week to Noguchi Memorial Institute for Medical Research (NMIMR) TB Laboratory for analysis.

\subsection{Mycobacterial Identification and Drug Susceptibility Testing (DST)}

Preliminary identification was made by the detection of acid fast bacilli (AFBs) in sputum samples by smear examination, using the Ziehl-Neelsen (ZN) staining technique. Subsequently, sputum samples were processed according to WHO guidelines [17]. Briefly, decontaminated sputum samples were centrifuged at $3000 \mathrm{~g}$ for 15 minutes and the supernatant discarded. The sediments obtained 
Table 1. Selected health facilities $(\mathrm{N}=67)$ and number of isolates obtained $(\mathrm{N}=503)$.

\begin{tabular}{|c|c|c|}
\hline Region & Health facility & Number of isolates \\
\hline \multicolumn{3}{|c|}{ Northern sector } \\
\hline \multirow[t]{7}{*}{ Ashanti } & & 38 \\
\hline & 1. Komfo Anokye Teaching Hospital & \\
\hline & 2. Obuasi Government Hospital & \\
\hline & 3. Anglogold Ashanti Hospital, Obuasi & \\
\hline & 4. Bryant Mission Hospital, Obuasi & \\
\hline & 5. Agogo Mission Hospital & \\
\hline & 6. Manyhia Hospital & \\
\hline \multirow[t]{10}{*}{ Brong Ahafo } & & 23 \\
\hline & 7. Sunyani Regional Hospital & \\
\hline & 8. Duayaw Nkwanta Catholic Hospital & \\
\hline & 9. Berekum Hospital & \\
\hline & 10. Dormaa Ahenkro Hospital & \\
\hline & 11. Goaso Hospital & \\
\hline & 12. Hwiediem Hospital & \\
\hline & 13. Techiman Catholic Hospital & \\
\hline & 14. Kintampo Hospital & \\
\hline & 15. Nkoranza Hospital & \\
\hline \multirow[t]{6}{*}{ Northern } & & 42 \\
\hline & 16. Tamale Teaching Hospital & \\
\hline & 17. Baptist Medical Center, Nalerigu & \\
\hline & 18. Saboba Hospital & \\
\hline & 19. Salaga Hospital & \\
\hline & 20. Yendi Hospital & \\
\hline \multirow[t]{6}{*}{ Upper West } & & 25 \\
\hline & 21. Wa Hospital & \\
\hline & 22. Nadowli Hospital & \\
\hline & 23. Jirapa Hospital & \\
\hline & 24. Nandom Hospital & \\
\hline & 25. Lawra Hospital & \\
\hline \multirow[t]{8}{*}{ Upper East } & & 50 \\
\hline & 26. Bolgatanga Hospital & \\
\hline & 27. Bawku Hospital & \\
\hline & 28. Navrongo Hospital & \\
\hline & 29. Sandema Hospital & \\
\hline & 30. Wiaga Hospital & \\
\hline & 31. Zebilla Hospital & \\
\hline & 32. Talensi Hospital & \\
\hline
\end{tabular}




\section{Continued}

\section{Southern sector}

Volta

33. Ho District Hospital

34. Ho Regional Hospital

35. Dzodze Catholic Hospital

36. Aflao Hospital

37. Amfoega Catholic Hospital

38. Kpando Mary Maiguate Hospital

Central

39. Winneba Hospital

40. Swedru Hospital

41. Cape Coast Regional Hospital

42. Cape Coast District Hospital

43. Ankaful Psychiatric Hospital

44. St Xavier Hospital, Assin Fosu

Eastern

45. Regional Hospital, Koforidua

46. St Joseph's Hospital, Koforidua

47. Nsawam Hospital

48. Atua Government Hospital

49. St Martin’s Hospital, Agomanya

50. Akuse Hospital

51. Holy Family Hospital, Nkawkaw

52. Atibie Government Hospital

Western

53. Effia Nkwanta Regional Hospital

54. Tarkwa Government Hospital

55. Eikwe Catholic Hospital

56. Axim Hospital

Greater Accra

57. Tema General Hospital

58. Tema Polyclinic

59. Tema Adom Clinic

60. 37 Military Hospital

61. La General Hospital

62. Maamobi Clinic

63. Kaneshie Polyclinic

64. Ussher Polyclinic

65. Achimota Hospital

66. Ridge Hospital

67. Korle-Bu Teaching Hospital 
were reconstituted with sterile phosphate buffer saline (PBS) and about 2 - 3 drops inoculated onto two slopes of Lowenstein-Jensen (LJ) media; one containing glycerol, the other containing $0.5 \%$ sodium pyruvate. The inoculated tubes were incubated at $37^{\circ} \mathrm{C}$ and monitored weekly for a maximum of 8 weeks for growth and subsequent isolation of mycobacterial species. Final identification of the species were based on acid fastness, colony morphology and ability to grow on LJ media containing $500 \mathrm{mg} / \mathrm{ml}$ p-nitrobenzoic acid (PNB) [18]. Line probe assay (LPA) was done to differentiate and determine isoniazid and rifampicin susceptibility profile of mycobacterial isolates from TB/HIV patients using Geno Type MTBC and Geno Type MTBDR plus (Hain Lifescience, Nehren Germany) respectively according to manufacturer's instructions [19].

\subsection{HIV Testing}

All patients were offered pre and post counselling for HIV test according to national guidelines [6]. Initial screening for HIV was done by eluting blood from filter paper and testing with First Response anti-HIV 1 \& 2 (Premier Medical Corporation, India). This test simultaneously detects and indicates specific antibodies against HIV-1 and HIV-2. Next, all HIV reactive samples were subjected to a line immunoblot assay, the Inno-Lia Score HIV-I \& II (Innogenetics, Belgium) to confirm the presence of HIV-1 and HIV-2 antibodies. Quality control of the HIV test was performed by random testing of $10 \%$ of HIV non-reactives from the rapid assay on the line immunoblot assay.

\subsection{Data Management and Analysis}

Raw data on-site were double entered into a data entry file-Microsoft Office Excel 2003 (Microsoft Excel, Palisade Corp, Newfield, NY, USA). Missing values, inconsistencies and outlier values were checked and corrected where necessary. Analysis of the data was done using Statistical Package for Social Sciences (SPSS) version 15.0 software for windows (SPSS Inc., Chicago, IL, USA). The threshold for statistical significance was $\mathrm{p} \leq 0.05$.

\subsection{Ethical Considerations}

The study was approved by the Scientific and Technical Committee and the Institutional Review Board of Noguchi Memorial Institute for Medical Research, Federal Wide Assurance 00001824 (NMIMR-IRB CPN 022/02-03). All patients involved in the study gave their informed consent prior to participation.

\section{Results}

Sixty seven health facilities from the northern and southern sectors of Ghana were involved in the study (Table 1). A total of 517 paired positive sputum samples were received from 517 patients. Out of this, a total of 503 TB patients [335 (66.6\%) males and 168 (33.4\%) females] had at least one culture positive sample (Table 1). Most patients, 136 (27.0\%) were within the age group 25 - 34 years ( $\mathrm{p}$ $<0.001)$. There were more new cases $468 / 503$ (93.0\%) than old cases $35 / 503$ 
$(7.0 \%)(\mathrm{p}<0.001)$. Majority $(73.6 \%), 370$ out of 503 were employed in the informal sector $(\mathrm{p}<0.001)$. The difference in HIV seroprevalence rates between males, 36/335 (10.75\%) and females, 38/168 (22.6\%) was statistically significant $(\mathrm{p}<0.05)$. The seroprevalence of HIV in newly diagnosed TB patients and those already on treatment, was $69 / 468(14.7 \%)$ and $5 / 35(14.3 \%)$ respectively $(\mathrm{p}>$ 0.05) (Table 2). Out of the 503 dried blood spots tested for HIV, 74 (14.7\%) were seropositive. This comprises 71 (14.1\%) HIV-1 and 3 (0.6\%) HIV-1 \& 2. None of the samples were positive for HIV-2 infection alone (Table 3). All the 503 isolates were Mycobacterium tuberculosis complex (MTBC) based on phenotypic identification. The LPA run for all 74 isolates from TB/HIV co-infected patients showed that 70 (94.6\%) were Mycobacterium tuberculosis while the remaining 4 (6\%) were Mycobacterium africanum (Table 4). Monoresistance to isoniazid and rifampicin were 4 (5.4\%) and 1 (1\%) respectively. However, resistance to both drugs (multi-drug resistant-MDR) was not observed. Sixty nine (93\%) isolates were susceptible to both drugs (Figure 1).

Table 2. Socio-demographic characteristics of patients whose culture were positive $(\mathrm{N}=$ 503).

\begin{tabular}{|c|c|c|c|c|}
\hline Variable & Frequency (\%) & $P$ value & HIV seroprevalence & $P$ value \\
\hline \multicolumn{5}{|l|}{ Sex } \\
\hline Male & $335(66.6)$ & $\mathrm{p}<0.001$ & 36 & $\mathrm{p}<0.05$ \\
\hline Female & $168(33.4)$ & & 38 & \\
\hline \multicolumn{5}{|l|}{ Age group (years) } \\
\hline$<15$ & $7(1.4)$ & & $\mathrm{p}<0.001$ & \\
\hline $15-24$ & $71(14.1)$ & & & \\
\hline $25-34$ & $136(27.0)$ & & & \\
\hline $35-44$ & $107(21.3)$ & & & \\
\hline $45-54$ & $111(22.1)$ & & & \\
\hline $55-64$ & $30(5.9)$ & & & \\
\hline $65+$ & $41(8.2)$ & & & \\
\hline \multicolumn{5}{|l|}{ TB Status } \\
\hline New case $\mathrm{a}^{\mathrm{a}}$ & $468(93.0)$ & $\mathrm{p}<0.001$ & 69 & $\mathrm{p}>0.05$ \\
\hline Old case ${ }^{b}$ & $35(7.0)$ & & 5 & \\
\hline \multicolumn{5}{|l|}{ Occupation } \\
\hline Informal $^{\mathrm{c}}$ & $370(73.6)$ & & & \\
\hline Formal $^{\mathrm{d}}$ & $26(5.2)$ & & & \\
\hline Unemployed $^{\mathrm{e}}$ & $42(8.3)$ & & & \\
\hline Student ${ }^{\mathrm{f}}$ & $65(12.9)$ & & & \\
\hline
\end{tabular}

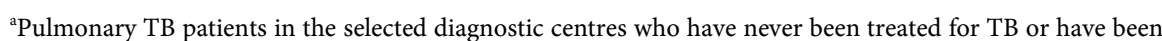
treated for a period less than one month. ${ }^{\mathrm{b}}$ All patients who have been previously treated for TB for a period more than one month. 'Jobs performed outside the formal structures that govern taxes, workplace regulations and social protection schemes. Jobs performed within formal structures that govern taxes, workplace regulations and social protection schemes. ${ }^{e}$ Not engaged in any gainful occupation. ${ }^{\text {fS }}$ Someone who attends an educational institution and not engaged in any gainful occupation. 
Table 3. HIV seroprevalence in 503 TB patients.

\begin{tabular}{cccccc}
\hline \multirow{2}{*}{ Region } & \multicolumn{2}{c}{${ }^{a}$ First Response } & \multicolumn{3}{c}{${ }^{b}$ Inno-Lia } \\
\cline { 2 - 6 } & HIV-1 & HIV-2 & HIV-1 & HIV-2 & HIV- 1\&2 \\
\hline Greater Accra & 12 & 0 & 12 & 0 & 0 \\
Eastern & 14 & 0 & 13 & 0 & 1 \\
Western & 8 & 0 & 8 & 0 & 0 \\
Central & 4 & 0 & 4 & 0 & 0 \\
Volta & 7 & 0 & 7 & 0 & 0 \\
Ashanti & 4 & 0 & 3 & 0 & 1 \\
Brong-Ahafo & 2 & 0 & 1 & 0 & 1 \\
Northern & 7 & 0 & 7 & 0 & 0 \\
Upper East & 10 & 0 & 10 & 0 & 0 \\
Upper West & 6 & 0 & 6 & 0 & 0 \\
TOTAL (\%) & $74(14.7)$ & $0(0)$ & $71(14.1)$ & $0(0)$ & $3(0.6)$ \\
\hline
\end{tabular}

${ }^{a}$ First Response-a rapid discriminatory assay for HIV-1 and HIV-2 antibodies. ${ }^{b}$ Inno-Lia-a line immunoblot assay used to confirm the presence of HIV antibodies in reactive HIV samples from initial rapid screening.

Table 4. Differentiation of MTBC isolates $(\mathrm{N}=74)$ from $\mathrm{TB} / \mathrm{HIV}$ co-infected participants using GenoType MTBC Assay.

\begin{tabular}{ccc}
\hline MTBC Species & Number & Percentage (\%) \\
\hline Mycobacterium tuberculosis & 70 & 94.6 \\
Mycobacterium africanum & 4 & 5.4 \\
\hline
\end{tabular}

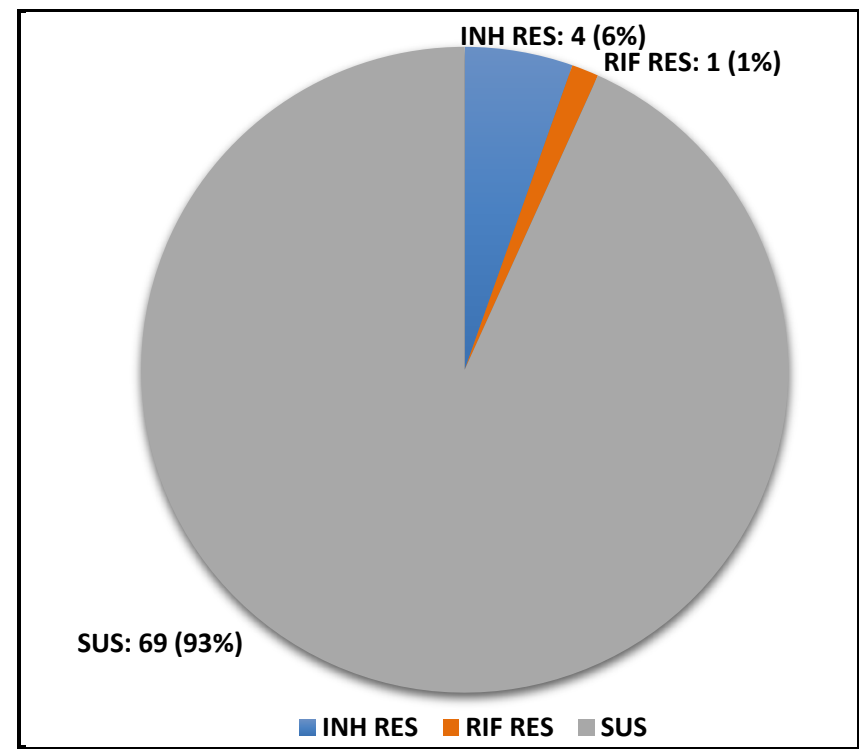

Figure 1. Drug Susceptibility Test (DST) profile of isolates $(\mathrm{N}=74)$ obtained from the TB/HIV co-infected participants. INH RES: isolates that are resistant to isoniazid. RIF RES: isolates that are resistant to rifampicin. SUS: isolates that are susceptible to both isoniazid and rifampicin. 


\section{Discussion}

TB/HIV co-infection is of great public health concern worldwide. This study sought to provide baseline data to inform health policies and actions to address this deadly disease duo in Ghana. Testing for HIV antibodies in TB patients as used in this study seemed an appropriate strategy to determine the burden of TB/HIV. While screening HIV-positive individuals for TB infection may be another option, HIV patients already burdened with stigmatisation may be unwilling to undertake a TB test as well. This assertion corroborates a report [15] showing that TB patients are more likely to accept HIV testing than HIV patients testing for TB. HIV prevalence in TB patients can be used as an indicator of the degree of spread of HIV in the general population. This information is also important for the provision of comprehensive HIV/AIDS care and support. $\mathrm{HIV}$ infection rates of up to $48 \%$ have been reported among newly diagnosed TB cases in Ghana [20], but the burden of TB among HIV-infected individuals in Ghana is barely acknowledged. Consistent with the global trend, more males (66.6\%) than females (33.4\%) were diagnosed with TB in this study [1]. The reasons for gender disparity with regards to TB infection is not well understood though it is believed that some cultural and biological factors may play a role [21]. Majority 70/74 (94.6\%) of the TB/HIV co-infected patients were infected with $M$. tuberculosis which is very similar to what other studies have reported in Ghana [22] [23]. On the contrary, the number of $M$. africanum (5.4\%) isolates was relatively low. Almost $93 \%$ of isolates obtained from TB patients were susceptible to both isoniazid and rifampicin. This result may be due to the fact that majority of the patients were new cases and as such had not taken any anti-TB drug before. The overall TB/HIV co-infection prevalence was $14.7 \%$ which was consistent with the $14.8 \%$ reported earlier in Ghana [9]. However, it was relatively lower than previous rates of $23.2 \%, 16.8 \%$ and $46.2 \%$ respectively reported in Ghana [12] [13] [14] as well as the average rate for Africa (32\%) [24]. These previous studies from Ghana were conducted in a very limited number of sites and the study population may not exactly reflect the general population. In Ghana infection with HIV-1 is most common, accounting for over $90 \%$ with very few cases of mixed infection of both HIV-1 and HIV-2 reported [25]. Hence it was not surprising to find similar results in this present study. There is an indication that there has been a steady change in the prevalence of TB/HIV co-infection over the last decade. This change in co-infection prevalence might be the result of improvements in public awareness, better treatment regimens and a general decline in HIV prevalence in Ghana [7]. Conversely, TB prevalence in Ghana is reported to be about three times higher than the estimates from WHO prior to the conduct of a TB prevalence survey in 2013 [2]. This suggests that many people are at an increased risk of HIV infection which necessitates a continuous collaborative plan for TB and HIV control activities to ensure comprehensive care for TB/HIV patients.

Some possible limitations in this study were that while TB patients will benefit from this study by knowing their HIV status and thus access treatment and care 
for both diseases, the same cannot be said for HIV patients who may be harbouring TB since the study focused on TB infected persons and not HIV infected persons. That notwithstanding, the results have been useful in strengthening the implementation of the joint TB/HIV strategy in Ghana. It has also been a vital biological marker of the HIV/AIDS epidemic in Ghana and indicating the impact on TB infection. Funds for the molecular testing part of the study became available in 2017 hence the delay in the publication of the findings.

\section{Conclusion}

The prevalence of TB/HIV co-infection was $14.7 \%$, and co-infection was more common in the sexually active age group of 25 - 34 years. Majority of these TB/HIV co-infected patients were infected with M. tuberculosis which were susceptible to both isoniazid and rifampicin. HIV-1 was the most common HIV strain infecting these patients. Further research is needed to monitor the trend and level of interaction between TB and HIV in Ghana.

\section{Acknowledgements}

We are thankful to the laboratory technologists of all the laboratories visited for sample collection and transportation. We also acknowledge the Global Fund against HIV/AIDS, TB \& Malaria and the Ghana Health Service for their financial support. The molecular analysis and publication was funded by DELTAS Africa Initiative [Afrique One-ASPIRE/DEL-15-008]. Afrique One-ASPIRE is funded by a consortium of donors including the African Academy of Sciences (AAS), Alliance for Accelerating Excellence in Science in Africa (AESA), the New Partnership for Africa's Development Planning and Coordinating (NEPAD) Agency, the Wellcome Trust [107753/A/15/Z] and the UK government.

\section{Competing Interest}

Authors declared that they have no competing interests.

\section{Authors' Contribution}

KKA and WKA came out with the study design and supervised the laboratory work. KKA contributed to the writing and editing of the manuscript. RO, CB and $\mathrm{NN}$ conducted the conventional TB laboratory work. JK and KB performed the HIV testing. SOA performed the molecular laboratory work, contributed to the manuscript writing and data analysis. GIM assisted with the proposal writing and data analysis. AH edited the study protocols. NAA and FAB contributed to the study implementation by providing reagents and equipment. All authors read and approved the final manuscript before submission.

\section{References}

[1] Kwan, C.K. and Ernst, J.D. (2011) HIV and Tuberculosis: A Deadly Human Syn- 
demic. Clinical Microbiology Reviews, 24, 351-376.

https://doi.org/10.1128/CMR.00042-10

[2] World Health Organization (2015) Global Tuberculosis Report 2015, Geneva, Switzerland.

http://apps.who.int/iris/bitstream/handle/10665/191102/9789241565059_eng.pdf;jse ssionid=A143550A79731FB650109B3FF068D215? sequence $=1$

[3] Selwyn, P.A., Hartel, D., Lewis, V.A., Schoenbaum, E.E., Vermund, S.H., Klein, R.S., et al. (1989) A Prospective Study of the Risk of Tuberculosis among Intravenous Drug Users with Human Immunodeficiency Virus Infection. New England Journal of Medicine, 320, 545-550. https://doi.org/10.1056/NEJM198903023200901

[4] Gao, J., Zheng, P. and Fu, H. (2013) Prevalence of TB/HIV Co-Infection in Countries Except China: A Systematic Review and Meta-Analysis. PLoS ONE, 8, e64915.

[5] Munsiff, S.S., Alpert, P.L., Gourevitch, M.N., Chang, C.J. and Klein, R.S. (1998) A Prospective Study of Tuberculosis and HIV Disease Progression. Journal of Acquired Immune Deficiency Syndromes and Human Retrovirology, 19, 361-366. https://doi.org/10.1097/00042560-199812010-00006

[6] Bonsu, F., Addo, N.A., Hesse, A., Kanlisi, N., Preko, P., Newman, M., et al. (2007) Implementation of HIV/TB Collaborative Activities for Ghana: Technical Policy and Guidelines. Ghana Health Service, Accra.

[7] Ghana AIDS Commission (2014) Ghana AIDS Commission Status Report 2014, Accra-Ghana.

[8] Narain, J.P. and Ying-Ru, L. (2004) Epidemiology of HIV-TB in Asia. Indian Journal of Medical Research, 120, 277-289.

[9] Ghana National Tuberculosis Programme (2005) Ghana National Tuberculosis Programme Annual Report 2005, Accra-Ghana.

[10] Ghana National Tuberculosis Programme (2006) Ghana National Tuberculosis Programme Annual Report 2006, Accra-Ghana.

[11] Slutsker, M. (2016) From Policy to Practice: How the TB-HIV Response Is Working. ACTION Global Health Advocacy Partnership. http://www.action.org

[12] Frimpong, E.H., Lawn, P., Dwemoh, B., Afful, B. and Acheampong, J.W. (1997) HIV Infection in Tuberculosis Patients in Kumasi, Ghana. Ghana Medical Journal, 31b, 850-854.

[13] Hesse, I.F.A. and Neequaye, A.R. (2003) HIV Infection in Pulmonary Tuberculosis Patients Admitted to the Korle Bu Teaching Hospital, Accra, Ghana. Ghana Medical Journal, 37, 7-11.

[14] Adjei, A.A., Adiku, T.K., Ayeh-Kumi, P.F. and Hesse, I.F. (2006) Prevalence of Human Immunodeficiency Virus Infection among Tuberculosis Suspect Patients in Accra, Ghana. West African Journal of Medicine, 25, 38-41.

[15] World Health Organization (2004) Guidelines for HIV Surveillance among Tuberculosis Patients. Second Edition, Geneva, Switzerland.

http://apps.who.int/iris/bitstream/handle/10665/68641/WHO_HTM_TB_2004.339. pdf? sequence $=1$

[16] Study Specific Procedure-SS-ECP-01 (001) LO (2008) Collection of Dried Blood Spots for the Nationwide Survey of TB/HIV Co-Infection in Ghana. NMIMR, Legon.

[17] World Health Organization (1998) Laboratory Services in Tuberculosis Control: Culture. Part III, Geneva.

http://apps.who.int/iris/bitstream/10665/65942/3/WHO_TB_98.258_(part3).pdf 
[18] Kent, P.T. and Kubica, G.P. (1985) Public Health Mycobacteriology: A Guide for the Level III Laboratory. United States Department of Health and Human Services, Centers for Disease Control, Atlanta.

[19] Mycobacteria Diagnostics: Hain Lifesciences, Nehren, Germany. https://www.hain-lifescience.de/en/home/competence/mykobakterien.html

[20] Ansa, G.A., Walley, J.D., Siddiqi, K. and Wei, X. (2014) Delivering TB/HIV Services in Ghana: A Comparative Study of Service Delivery Models. Trans Royal Society Tropical Medicine Hygiene, 108, 560-567. https://doi.org/10.1093/trstmh/tru110

[21] Esmael, A., Tsegaye, G., Wubie, M. and Endris, M. (2013) Tuberculosis and Human Immune Deficiency Virus Co-Infection in Debre Markos Referral Hospital in Northwest Ethiopia: A Five Years Retrospective Study. Journal of AIDS Clinical Research, 4, 263. https://doi.org/10.4172/2155-6113.1000263

[22] Addo, K.K., Owusu-Darko, K., Yeboah-Manu, D., Caulley, P., Minamikawa, M., Bonsu, F., et al. (2007) Mycobacterial Species Causing Pulmonary Tuberculosis at the Korle Bu Teaching Hospital, Accra. Ghana Medical Journal, 41, 52-57.

[23] Yeboah-Manu, D., Asante-Poku, A., Bodmer, T., Stucki, D., Koram, K., Bonsu, F., et al. (2011) Genotypic Diversity and Drug Susceptibility Patterns among M. tuberculosis Complex Isolates from South-Western Ghana. PLOS ONE, 6, e21906.

[24] Gray, J.M. and Cohn, D.L. (2013) Tuberculosis and HIV Co-Infection. Seminars in Respiratory and Critical Care Medicine, 34, 32-43. https://doi.org/10.1055/s-0032-1333469

[25] Ghana: UNAIDS/WHO (2004) Epidemiological Fact Sheet on HIV/AIDS and Sexually Transmitted Infections.

http://data.unaids.org/publications/fact-sheets01/ghana_en.pdf 


\section{List of Abbreviations}

AFB: Acid fast bacilli

AIDS: acquired immune deficiency syndrome

A-LYS: Alcalic lysis

Anti-TB: Anti-tuberculosis

CPC: Cetylpyridinium Chloride

DOTS: Directly Observed Treatment Short course

DST: Drug susceptibility testing

HIV: Human Immunodeficiency Virus

LJ: Lowenstein-Jensen

LPA: Line probe assay

MDR: Multi-drug resistance

MTBC: Mycobacterium tuberculosis complex

NMIMR: Noguchi Memorial Institute for Medical Research

TB: Tuberculosis

PBS: Phosphate buffer saline

PCR: Polymerase chain reaction

PNB: Para-Nitrobenzoic acid

WHO: World Health Organization

ZN: Ziehl-Neelsen 